\title{
LINFOMA PRIMARIO DEL SISTEMA NERVIOSO CENTRAL COMO PRIMERA MANIFESTACIÓN DE SIDA. PRESENTACIÓN DE UN CASO Y REVISIÓN DE
} LA LITERATURA

\author{
Marcelo Corti ${ }^{1,2}$, María F. Villafañe ${ }^{1,2}$, Osvaldo Franze ${ }^{3}$, Ana Campitelli $^{4}$ y \\ Marina Narbaitz ${ }^{5}$
}

RESUMEN

El linfoma primario del sistema nervioso central (LPSNC) es una neoplasia infrecuente que comprende solo el $5 \%$ del total de los linfomas de localización extranodal. El LPSNC se define como un linfoma de tipo No Hodgkin (LNH) que solo infiltra el eje cerebro espinal sin compromiso sistémico. Esta neoplasia es más frecuente en sujetos con inmunodeficiencias congénitas y adquiridas. En los pacientes con SIDA, el LPSNC ocurre con igual incidencia en todos los grupos de riesgo y edades pero con una mayor prevalencia en aquellos con recuentos de linfocitos T CD4 + de $<$ de 50 células $/ \mu \mathrm{L}$. Se trata de un linfoma de células grandes, de alto grado de malignidad, y fuertemente asociado en su patogenia al virus de Epstein Barr (VEB) cuyo genoma se detecta en las células linfoides atípicas. La presencia del genoma del VEB se detecta en el $100 \%$ de los casos de LPSNC asociado al SIDA pero esto no ocurre en otras clases de pacientes con esta misma neoplasia. En pacientes con SIDA y lesiones de masa cerebral ocupante, la detección del genoma del VEB en el líquido cefalorraquídeo (LCR) a través de la reacción en cadena de la polimerasa (PCR) tiene una sensibilidad del $83 \%$ a $100 \%$ y una especificidad $>$ del $90 \%$ para el diagnóstico. El uso difundido de la terapia antirretroviral de gran actividad (TARGA) se asoció con una declinación en la incidencia de LPSNC. El pronóstico de esta neoplasia en pacientes con SIDA es pobre y se asocia con una mediana de supervivencia de 2 a 3 meses en la era previa a la TARGA con una discreta prolongación en la supervivencia con la TARGA. Se presenta un paciente que desarrolló un LPSNC como primera manifestación de SIDA y se efectúa una actualización de la literatura sobre el tema.

PALABRAS CLAVE: Linfoma primario del sistema nervioso central; SIDA; VIH.

\footnotetext{
1. División "B”, HIV/SIDA, Hospital de Enfermedades Infecciosas F. J. Muñiz, Buenos Aires, Argentina.

2. Departamento de Medicina, Orientación de Enfermedades Infecciosas, Facultad de Medicina, Universidad de Buenos Aires, Argentina.

3. Servicio de Neurocirugía, Hospital de Enfermedades Infecciosas F. J. Muñiz, Buenos Aires, Argentina.

4. Laboratorio de Anatomía Patológica, Hospital de Enfermedades Infecciosas F. J. Muñiz, Buenos Aires, Argentina.

5. Laboratorio de Anatomía Patológica, Academia Nacional de Medicina, Buenos Aires, Argentina.
}

Dirección para correspondencia: marcelocorti@fibertel.com.ar

Recibido para publicación en: 10/7/2015. Revisado en: 27/10/2015. Aceptado en: 17/11/2015. 


\begin{abstract}
Primary central nervous system lymphoma as first manifestation of AIDS. Report of a case and literature review

Primary central nervous system lymphoma (PCNSL) is an infrequent neoplasm comprising only $5 \%$ of extranodal lymphomas. PCNSL is defined as a non-Hodgkin's lymphoma (NHL) that is limited to the craniofacial axis without systemic involvement. This neoplasm is most common in patients with congenital and acquired immunodeficiencies in comparison with the general population. In AIDS patients, PCNSL occurs with a similar incidence in all the risks groups and ages with a high incidence in individuals with a count of less than $50 \mathrm{CD} 4$ $\mathrm{T}$ cells $/ \mu \mathrm{L}$. This tumor is characteristically a large B-cell lymphoma strongly related to the presence of the Epstein Barr virus (EBV) genome in the atypical cells. The presence of EBV is detected in all AIDS-associated PCNSL but this finding is not observed in other patients with the same neoplasm. In AIDS patients with cerebral mass lesions, the detection of EBV in the cerebrospinal fluid (CSF) by DNA polymerase chain reaction (PCR) has a sensitivity of $83 \%$ to $100 \%$ and specificity higher than $90 \%$. The use of highly active antiretroviral treatment (HAART) is associated with a decline in the incidence of AIDS-related PCNSL. The prognosis of AIDS-associated PCNSL is generally poor with a median survival of two to three months in the pre HAART era and a mild improvement in overall survival in the HAART era. Here we describe a patient who developed a PCNSL as a first manifestation of AIDS and we performed a review of the literature.
\end{abstract}

KEY WORDS: Primary central nervous system lymphoma; AIDS; HIV.

\title{
INTRODUCCION
}

El linfoma primario del sistema nervioso central (LPSNC) es una neoplasia marcadora de sida que muestra una incidencia significativamente mayor en la población infectada por el virus de la inmunodeficiencia humana (VIH) en comparación con la población general.

El LPSNC es un linfoma no Hodgkin (LNH) que se caracteriza por su alto grado de malignidad e histológicamente por tratarse de un tumor de grandes células de fenotipo B (7). Se la define como una neoplasia limitada al SNC sin compromiso sistémico. Si bien desde la introducción de la terapia antirretroviral de gran actividad (TARGA) la incidencia de LPSNC ha disminuido, todavía es una complicación frecuente en aquellos sujetos que desconocen su condición de infectados por el retrovirus o en aquellos que no reciben TARGA o si lo hacen no tienen buena adherencia (3).

Se presenta un paciente que desarrolló un LPSNC como primera manifestación de sida y se revisa la literatura sobre el tema. 


\section{CASO CLINICO}

Paciente de 46 años que ingresa con cuadro de un mes de evolución compuesto por cefalea, fiebre de predominio vespertino, sudoración nocturna y pérdida de peso (10 Kg en dos meses). En la semana previa a la internación se agrega paresia faciobraquiocrural derecha. Al examen físico de ingreso se constatan cono datos de relevancia fiebre $38,5^{\circ} \mathrm{C}$, palidez cutáneo-mucosa, hemiparesia faciobraquiocrural derecha con hipotonía e hiporreflexia en ese hemicuerpo. El laboratorio de ingreso mostró hematocrito: $30 \%$, Hb: 9,2 g/\%, GB: $4.300 / \mathrm{mm}^{3}$ (83\% PMN, $10 \%$ linfocitos); plaquetas: $160.000 / \mathrm{mm}^{3}$,VSG: $70 \mathrm{~mm} 1^{a}$ hora; función renal y hepática conservadas, serología para Chagas, toxoplasmosis y VDRL negativas. La serología para VIH fue reactiva por técnica de ELISA de $4^{\circ}$ generación y confirmación por Western blot. El recuento de linfocitos T CD4+ fue de $0(0 \%)$ y la carga viral plasmática de 209.000 copias/ $\mu \mathrm{L}\left(\log _{10} 5,3\right)$. Se realizaron hemocultivos para gérmenes comunes, hongos y micobacterias que resultaron negativos. Se realizó tomografía computada de cerebro con contraste que mostró lesión hipodensa en región lentículocapsular y talámica izquierda sobre sustancia blanca periventricular, con realce periférico heterogéneo del contraste, edema perilesional y compresión con colapso del sistema ventricular homolateral. (Figuras 1 y 2 ).
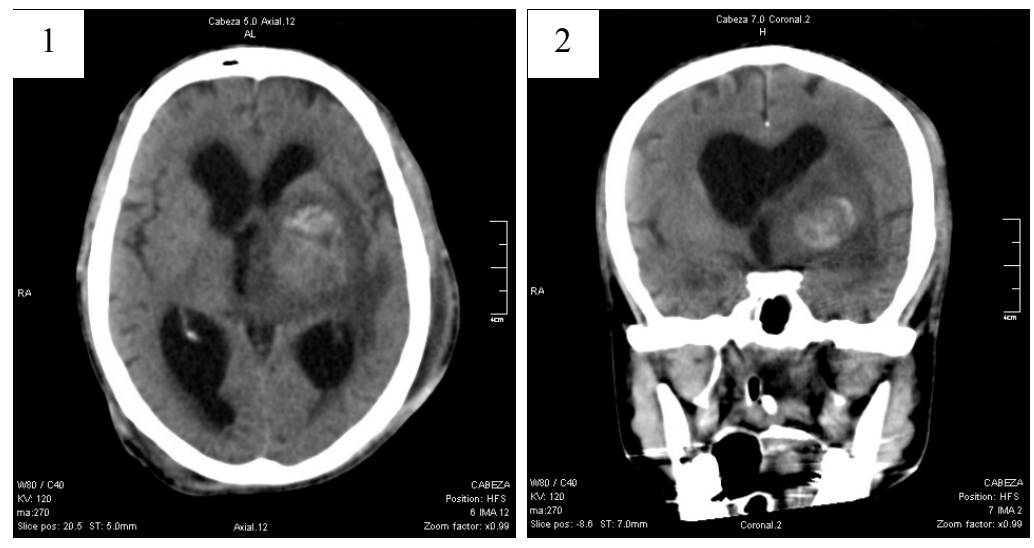

Figura 1: TC de cerebro corte axial: lesión hipodensa en región lentículocapsular y talámica izquierda sobre sustancia blanca periventricular, con realce periférico heterogéneo del contraste.

Figura 2: TC de cerebro plano coronal: lesión periventricular con realce del contraste, gran edema perilesional y colapso del ventrículo homolateral. 
$\mathrm{Al}$ conocerse su condición serológica para VIH se inicia tratamiento empírico para toxoplasmosis cerebral en base a pirimetamina, clindamicina, ácido folínico, corticoides y anticonvulsivos a las dosis habituales. Se efectuó punción lumbar; el líquido cefalorraquídeo (LCR) no mostró alteraciones bioquímicas en su composición; los estudios microbiológicos para gérmenes comunes, BAAR y hongos resultaron negativos. La PCR cuantitativa resultó negativa para VJC, para los neuroherpevirus CMV, HSV 1 y 2; VZV y HHV-6 y positiva para virus de Epstein-Barr (VEB) con una carga viral de 28.870 copias/ $\mathrm{mL}\left(\log _{10}: 4.47\right)$. Tras dos semanas de tratamiento empírico antiparasitario sin respuesta clínica ni radiológica, se realizó biopsia cerebral esterotáctica. El examen directo y los cultivos de las muestras obtenidas fueron negativos para gérmenes comunes, BAAR y hongos. El estudio histopatológico mostró, a menor aumento, un fragmento de sustancia blanca con infiltrados de células linfoides atípicas, con disposición predominantemente perivascular (Figura 3). A mayor aumentó se observó una proliferación de células de gran diámetro, con núcleos irregulares, macro y anisocarióticos, nucléolos prominentes y citoplasma variable, que se disponen de manera intersticial y perivascular (Figura 4). Con técnicas de inmunomarcación, las células descriptas expresan CD20 con co-expresión de MUM1, siendo negativas para CD3, CD5 y Bcl6. El índice de proliferación Ki67 resultó elevado (70\%). El diagnóstico histopatológico final fue de linfoma difuso de grandes células B, de alto grado de agresividad histológica, primario del SNC. La biopsia de medula ósea no mostró infiltración por células atípicas.

El paciente evolucionó de manera desfavorable, empeorando rápidamente su condición clínica y neurológica, sin posibilidades de implementar ningún tratamiento específico. Falleció por cuadro séptico con falla multiorgánica a los 2 meses de efectuado el diagnóstico de la neoplasia.
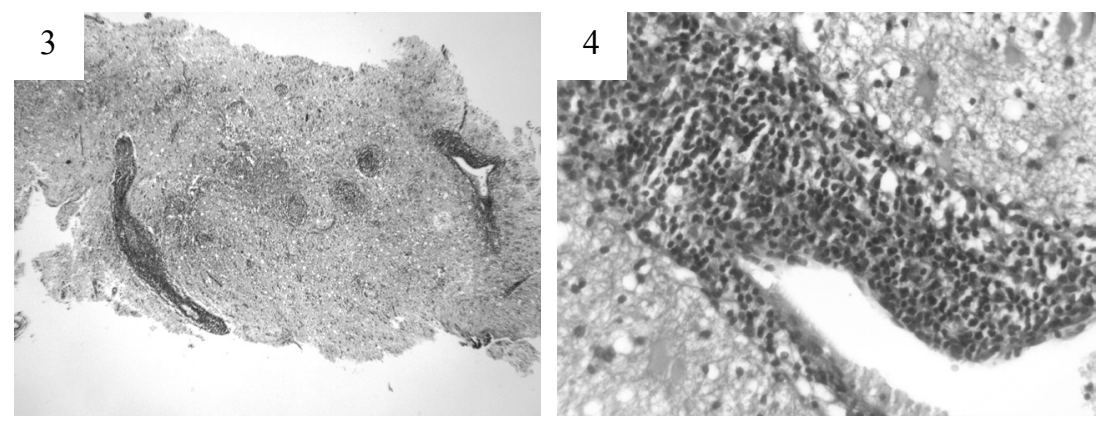

Figura 3: Fragmento de sustancia blanca con infiltrados de células linfoides atípicas de disposición difusa y predominantemente perivascular (H\&E 40x). Figura 4: Proliferación de células linfoides de gran tamaño, con núcleos pequeños y varios nucléolos, dispuestas rodeando un vaso (H\&E 400x). 


\section{DISCUSION}

Los pacientes con enfermedad VIH/sida avanzada y recuento de linfocitos $\mathrm{T}$ CD4 + de $<$ de 50 cél $/ \mu \mathrm{L}$ presentan con mucha frecuencia complicaciones neurológicas vinculadas con infecciones oportunistas y tumores. El LPSNC se debe incluir en el diagnóstico diferencial de las lesiones de masa cerebral ocupante en los pacientes con sida (11). La tomografía computarizada (TC) y la resonancia magnética (RM) son los métodos de imágenes más utilizados para el abordaje inicial de los pacientes VIH positivos con lesiones de masa cerebral ocupante. Clásicamente esta neoplasia se presenta como una lesión ocupante de espacio, generalmente única que refuerza con el contraste, tanto en la TC como en la RM. La localización es por lo general supratentorial, aunque puede comprometer también el cerebelo y las estructuras del tronco encefálico (7). Los estudios de neuroimágenes permiten sospechar el diagnóstico que solo se confirmará por el estudio histopatológico de las muestras obtenidas mediante biopsia estereotáctica (6). La TC muestra por lo general lesiones únicas, de gran tamaño $(>2 \mathrm{~cm})$, que refuerzan con el contraste en forma homogénea y se localizan en la sustancia blanca periventricular (5). Sobrido Sampedro y col. (14) en un estudio retrospectivo y descriptivo que incluyó 21 pacientes inmunodeprimidos $(52,4 \%$ VIH positivos) con LPSNC tuvieron un $61 \%$ de lesiones únicas y un $85,7 \%$ de lesiones supratentoriales. En este mismo estudio, un $52,4 \%$ de las lesiones fueron heterogéneas en la RM, un 57,1\% evidenció contornos bien definidos y el tamaño fue en promedio $>$ de $3 \mathrm{~cm}$. En cuanto a la TC, el $60 \%$ de las lesiones fueron hipodensas y un $91 \%$ tuvieron realce periférico del contraste. Dina (8) en un estudio retrospectivo, señala que las lesiones cerebrales focales con refuerzo del contraste y prolongación ependimaria y subependimaria en las neuroimágenes son muy sugerentes de LPSNC. El número, el tamaño y la localización de las lesiones son los signos radiológicos de mayor valor para el diagnóstico diferencial entre LPSNC y toxoplasmosis cerebral, que es la infección oportunista que con más frecuencia produce lesiones de masa ocupante en los pacientes con sida.

Existen otros métodos de diagnóstico por imágenes que pueden ayudar al diagnóstico diferencial entre LPSNC y otras masas ocupantes cerebrales de naturaleza infecciosa. Actualmente, las técnicas de avanzada por RM (como la perfusión, espectroscopia o difusión) permiten establecer un diagnóstico más preciso, acortando el diagnóstico diferencial, sobre todo en lesiones con comportamiento atípico. Respecto a las imágenes de RM por difusión, el grado de restricción a la difusión es directamente proporcional a la celularidad tisular (en la imagen potenciada por difusión, el LPSNC presenta una difusión restringida con un mapa de coeficiente de difusión aparente disminuido, más bajo que los valores de los gliomas de alto grado y las metástasis) $(10,14)$. Por su parte, la perfusión por RM refleja la existencia de angiogénesis. El LPSNC, en el mapa del volumen sanguíneo cerebral relativo, presenta un valor de perfusión reducido en comparación con las neoplasias de alto grado o metástasis, pero es más elevado 
que el de los procesos infecciosos (por ejemplo, el de la toxoplasmosis) $(10,14)$. La espectroscopia con RM es un método no invasivo que ofrece información metabólica sobre el cerebro. La mayoría de los LPNSC presentan un incremento en los picos de lípidos y lactato con una relación colina/creatina elevada (hallazgo que comparte con el glioblastoma multiforme y las metástasis, pero que ayuda a diferenciarlo de otras entidades) (10). Finalmente, la tomografía computarizada con emisión de fotón único (SPECT) muestra una rápida y marcada captación del radioisótopo por el LPSNC y no por aquellas lesiones de naturaleza infecciosa o inflamatoria. Antinori y col.(2) comprobaron que SPECT con talio 201 tiene una sensibilidad del $92 \%$ y un valor predictivo positivo del $94 \%$ para el diagnóstico de LPSNC. Las causas de resultados falsos positivos de esta técnica incluyen criptococomas, tuberculomas y abscesos bacterianos (12).

El VEB se encuentra fuertemente asociado con la patogenia del LPSNC (13). La detección del DNA del VEB en el LCR de pacientes VIH positivos con lesiones cerebrales sugerentes de LPSNC es una prueba diagnóstica sensible y específica (9). Además, el genoma del VEB ha sido detectado en el LCR de pacientes VIH positivos sin lesiones cerebrales pero que posteriormente desarrollaron LPSNC por lo que se lo ha propuesto también como un potencial predictor para el futuro desarrollo de esta neoplasia $(1,4)$.

El tratamiento actual de todos los pacientes VIH positivos con linfomas, sean estos de tipo Hodgkin o no Hodgkin, incluye la combinación de la TARGA y la quimioterapia. La profilaxis para las infecciones oportunistas debe implementarse en aquellos pacientes que reciben quimioterapia aún con recuentos de linfocitos T CD4 + > 200 cél $/ \mu \mathrm{L}$ (15). El tratamiento de los LPSNC ha experimentado algunos cambios en los últimos años que han permitido modificar parcialmente el pronóstico de esta clase de pacientes. En este sentido, los mejores resultados se han obtenido con el uso de TARGA, corticoides y metotrexato, con o sin radioterapia sobre el eje cerebroespinal. Sin embargo, el pronóstico continúa siendo pobre; sin tratamiento, la supervivencia es de 1 a 3 meses y se incrementa a 3 a 18 meses con el tratamiento señalado (15).

Hay muchas razones, individuales y de salud pública, para promover y facilitar un diagnóstico precoz de la infección por el VIH y ello es una responsabilidad de todo el personal de salud, independientemente de su especialidad. Los profesionales médicos deben ofrecer y facilitar el acceso a las pruebas de diagnóstico de la infección por el retrovirus y practicarlos salvo que haya una oposición explícita por parte del paciente.

De esta manera podría evitarse que muchos pacientes lleguen a la consulta y al diagnóstico en estadios avanzados de la inmunodeficiencia como en el caso que se describe.

En este aspecto, el inicio precoz del tratamiento antirretroviral resulta beneficioso para reducir los reservorios no únicamente en personas con primoinfección sino también en aquellas con infección en fase crónica. Diversos estudios han demostrado que el inicio temprano de la TARGA puede ayudar a 
prevenir el deterioro del sistema inmunológico y alejar a los pacientes del riesgo de desarrollar infecciones oportunistas graves o neoplasias que incrementan notablemente la morbimortalidad de esta población de pacientes como ocurrió en el caso que se presenta.

\section{REFERENCIAS}

1. Al-Shahi R, Bower M, Nelson MR, Gazzard BG. Cerebrospinal fluid Epstein-Barr virus detection preceding HIV-associated primary central nervous system lymphoma by 17 months. J Neurol 247: 471-472, 2000.

2. Antinori A, De Rossi G, Ammassari A, Cingolani A, Murri R, Di Giuda D, De Luca A, Pierconti F, Tartaglione T, Scerrati M, Larroca LM, Ortona L. Value of combined approach with thallium-201 single emission computed tomography and Epstein-Barr virus DNA polymerase chain reaction in CSF for the diagnosis of AIDS-related primary CNS lymphoma. J Clin Oncol 17: 554-560, 1999.

3. Bower M, Powles T, Nelson M, mandalia S, Gazzard B, Stebbing J. Highly active antiretroviral therapy and human immunodeficiency virus-associated primary cerebral lymphoma. $J$ Natl Cancer Inst 98: 1088-1091, 2006.

4. Cinque P, Vago L, Dahl H, Brytting M, Terreni MR, Fornara C, Racca S, Castagna A, Monforte AD, Wahren B, Lazzrin A, Linde A. Polymerase chain reaction on cerebrospinal fluid for diagnosis of virus-associated opportunistic diseases of the central nervous system in HIV-infected patients. AIDS 10: 951-958, 1996.

5. Ciricillo SF, Rosemblum ML. Use of CT and MR imaging to distinguish intracranial lesions and to define the need for biopsy in AIDS patients. J Neurosurg 73: 720-724, 1990.

6. Corti M, Metta H, Villafañe MF, Yampolsky C, Schtirbu R, Sevlever G, Garrido D. La biopsia estereotáctica en el diagnóstico de las lesiones cerebrales focales en sida. Medicina (B. Aires) 68 : 285-290, 2008.

7. Corti M, Villafañe F, Trione N, Schtirbu R, Yampolsky C, Narbaitz M. Primary central nervous system lymphomas in AIDS patients. Enferm Infecc Microbiol Clin 22: 332-336, 2004.

8. Dina TS. Primary central nervous system lymphoma versus toxoplasmosis in AIDS. Radiology 179: 823-828, 1991.

9. Flinn IW, Ambinder RF. AIDS primary central nervous system lymphoma. Curr Opin Oncol 8: 373-376, 1996.

10. Haldorsen IS, Espeland A, Larsson EM. Central nervous system lymphoma: characteristic findings on traditional and advanced imaging. Am J Neuroradiol 32: 984-992, 2011.

11. Ivers LC, Kim AY, Sax PE. Predictive value of polymerase chain reaction of cerebrospinal fluid for detection of Epstein-Barr virus to establish the diagnosis of HIV-related primary central nervous system lymphoma. Clin Infect Dis 38: 1629-1632, 2004.

12. Sanjaume M, Rodríguez-Carballeira E, Martinez-Lacasa J, García J, Garau J. SPECT cerebral con talio-201 en el diagnóstico diferencial de lesiones cerebrales focales en pacientes con sida. Enferm Infec Microbiol Clin 15: 28-31, 1997.

13. Segura MJ, Miranda MA, DiLorenzo G, Garau ML, Derin H. Recomendaciones para el manejo de pacientes HIV+ con masas intracraneanas en nuestro medio. Rev Neurol Arg 24: 36-42, 1999.

14. Sobrido Sampedro C, Corroto JD, Arias González M, Iglesias Castañón A, Corroto Murua J, Pumar Cebreiro JM. Neuroimagen del linfoma primario del sistema nervioso central en pacientes inmunodeprimidos. Rev Argent Radiol 78: 5-12, 2014.

15. Valencia Ortega ME. AIDS-related malignancies. A new approach. AIDS Rev 10: 125-127, 2008. 